\title{
Direcciones futuras de la ecotoxicología en Chile: implicancias para la evaluación de riesgo ambiental de productos veterinarios utilizados en acuicultura
}

\author{
Future directions of Chilean ecotoxicology: Implications for the environmental risk \\ assessment of veterinary products used in aquaculture
}

MATÍAS H. MEDINA ${ }^{1,2, *}$ \& RODRIGO RAMOS-JILIBERTO ${ }^{3,4}$

\author{
${ }^{1}$ AVS Chile, Imperial 0655, Of. $3^{\text {a }}$, Puerto Varas, Chile \\ ${ }^{2}$ Centro i-mar, Universidad de Los Lagos, Camino Chinquihue km 6, Puerto Montt, Chile \\ ${ }^{3}$ Departamento de Ciencias Ecológicas, Facultad de Ciencias, Universidad de Chile, casilla 653 Santiago, Chile \\ ${ }^{4}$ Centro Nacional del Medio Ambiente. Fundación de la Universidad de Chile. Av. Larraín 9975, La Reina, Santiago, Chile \\ * Autor correspondiente: matias.medina@avs-chile.cl
}

\begin{abstract}
RESUMEN
La relación bidireccional de influencia que la acuicultura establece con el ambiente en el cual se desarrolla determina límites al crecimiento de esta actividad económica en Chile. Esto se ha traducido tanto en restricciones técnicas, como en conflictos ambientales y sociales. La creciente demanda de nuevos productos quimioterapéuticos y su consecuente vertido en ambientes acuáticos es considerado uno de los principales problemas ambientales que enfrenta la industria del salmón en Chile. Sin embargo, poco se sabe acerca de los efectos que ejercen estas sustancias sobre especies no objetivo y, en general, sobre la estructura y función de los ecosistemas expuestos. Mediante la aplicación de teoría y métodos de ecología contemporánea, el trabajo aquí presentado busca contribuir oportunamente al desarrollo de una metodología que permita reducir el nivel de incertidumbre asociado a la evaluación de riesgo ambiental de productos veterinarios utilizados en acuicultura. Se revisa el estado actual de la evaluación de riesgo ambiental en Chile y se identifican aspectos críticos y factibles de corregir en el marco de los procedimientos utilizados. Finalmente, se entrega una propuesta metodológica general que contempla extender de un modo coherente las pruebas ecotoxicológicas estándar, combinando experimentación a niveles comunitario, poblacional e individual, junto a la modelización matemática de sistemas ecológicos.
\end{abstract}

Palabras clave: ecología aplicada, gestión ambiental, mesocosmos, microcosmos, sistemas complejos.

\begin{abstract}
The bidirectional relationship that aquaculture has with the environment set limits to the growth this economic activity has in Chile. This condition has generated both technical constraints and environmental as well as social conflicts. The growing demand for new chemotherapeutic compounds and their release into aquatic environments is now considered one of the main problems being faced by the salmon industry in Chile. However, little is known about the effects these substances exert on non-target species and on the structure and functioning of exposed ecosystems. Through the application of theory and methods from contemporary ecology, the present work aims at contributing towards the development of a methodology that allows a reduction of the uncertainty level associated to the environmental risk assessment of veterinary products used in aquaculture. The current status of the environmental risk assessment in Chile is revised and those critical aspects feasible to fix are identified, considering the procedures currently in use. Finally, a general methodological approach is proposed that considers a coherent expansion of standard ecotoxicological tests combining research at community, population, and individual levels, with mathematical modeling of ecological systems.
\end{abstract}

Key words: applied ecology, complex systems, environmental management, mesocosms, microcosms. 


\section{INTRODUCCIÓN}

La salmonicultura es hoy en día la actividad con mayor producción y valor de exportación en la acuicultura chilena. Por su influencia en diferentes sectores productivos del país, esta industria se ha transformado en un importante factor en la diversificación de la economía nacional y un pilar fundamental en la estrategia orientada a transformar a Chile en una potencia mundial en materia de alimentos.

Sin embargo, su crecimiento ha comenzado a enfrentar problemas técnicos, sociales y medioambientales que limitan su expansión. En el último tiempo y luego de un largo periodo de crecimiento sostenido, la industria ha comenzado a mostrar una reducción significativa en su producción. Esta situación se debe en gran medida a la relación bidireccional de influencia que la acuicultura establece con el ambiente en el cual se desarrolla (Acuicultura $\leftrightarrow$ Ambiente), donde (i) el ambiente de cultivo puede afectar el desempeño biológico de los organismos cultivados, generando una situación sanitaria desfavorable como resultado del estrés y las enfermedades asociadas a una mala calidad del agua (Acuicultura $\leftarrow$ Ambiente) y (ii) la producción de organismos altera la calidad de su ambiente, lo cual genera tanto preocupación como reacciones en la sociedad en general (Acuicultura $\rightarrow$ Ambiente). En general, la acuicultura del salmón ha sido asociada con los siguientes impactos ambientales: (i) aporte de nutrientes y materia orgánica al ecosistema, proveniente del alimento y las heces de los peces en cultivo, (ii) escape de los peces, (iii) liberación de compuestos químicos utilizados en la prevención de fouling y (iv) liberación de productos veterinarios (i.e. biocidas, quimioterapéuticos, antibióticos) utilizados en el control de parásitos y otras enfermedades (Gowen \& Bradbury 1987, Hallaegraeff 1993, Folke et al. 1998, Goldburg \& Naylor 2005).

En Chile, los mismos potenciales efectos han sido descritos en asociación con el desarrollo de la acuicultura (Buschmann \& Fortt 2005). Sin embargo, la posible correlación entre un aumento en la salmonicultura y un incremento en el número de floraciones algales nocivas (Buschmann et al. 2006), así como de infestaciones por ecotoparásitos en poblaciones de peces nativos
(Sepúlveda et al. 2004), son aspectos de especial relevancia para la zona sur del país. Debido a la situación sanitaria adversa que actualmente afecta a la industria del salmón en Chile, el vertido de productos veterinarios al ambiente acuático representa también uno de los principales problemas que esta ha debido enfrentar, destacándose el elevado uso profiláctico de antibióticos (Cabello 2004) y la aplicación de quimioterapéuticos para el control de ectoparásitos (e.g. Bravo et al. 2008). Aunque es de preocupación pública, prácticamente nada se sabe acerca del efecto que ejercen estas sustancias sobre especies no objetivo $\mathrm{y}$, en general, sobre la estructura y funcionamiento de los ecosistemas expuestos (Soto \& Norambuena 2004, Buschmann et al. 2006, León-Muñoz et al. 2007, Costa-Pierce et al. 2008, Burridge et al. 2008). Esta situación ha estimulado y profundizado los permanentes conflictos ambientales que surgen entre la industria acuícola nacional, ONGs y la sociedad en general (Reel 2007, Barrionuevo 2008).

Si Chile desea mantener un crecimiento sustentable de la industria del salmón y consolidar así el cluster de acuicultura definido por el Consejo Nacional de Innovación (CNIC 2007), esta falta de conocimiento es un problema que debe ser solucionado en un futuro cercano. En relación a esto, la evaluación de riesgo ambiental de productos veterinarios solicitada por el Instructivo Técnico para el Registro de Productos Farmacológicos (I-PP-MR-002, 2007) elaborado por el Servicio Agrícola y Ganadero (SAG) ha cobrado especial interés para la industria del salmón en Chile. Lo anterior se explica principalmente por dos razones; (i) la creciente necesidad de disponer de una mayor variedad de productos farmacológicos para el control de distintas enfermedades, y (ii) la dificultad que han experimentado las empresas farmacéuticas proveedoras en el proceso de registro de nuevos productos.

Este trabajo pretende contribuir oportunamente al desarrollo de una metodología que permita reducir el nivel de incertidumbre asociado a las evaluaciones de riesgo ambiental realizadas en el marco del registro de productos veterinarios para la acuicultura. Mediante la revisión de los procedimientos vigentes así como del análisis 
de ejemplos específicos, este trabajo expone y discute diferentes debilidades identificadas en la metodología que solicita el instructivo y propone formas alternativas de evaluación, basadas en la teoría y métodos de la ecología contemporánea. Las propuestas presentadas apuntan a obtener la evidencia necesaria para revelar la posible ocurrencia de efectos causantes de cambios en la estructura, dinámica y funcionamiento del ecosistema expuesto.

\section{Evaluación de riesgo ambiental en Chile}

La contaminación antropogénica de ambientes naturales puede producir efectos negativos en organismos y poblaciones de diferentes taxa, generando cambios en la estructura, dinámica y funcionamiento tanto de las comunidades como de los ecosistemas de los cuales forman parte (Parker et al. 1999). Dependiendo de la intensidad, duración y frecuencia de las descargas de contaminantes, estos cambios resultan de: (i) la eliminación de poblaciones sensibles debido a efectos tóxicos directos, (ii) el reemplazo de estas especies por otras tolerantes debido al debilitamiento de la competición por recursos, (iii) modificación de la magnitud de las interacciones ecológicas como resultado de la alteración de las tasas de consumo de recursos en aquellas especies sensibles a los contaminantes, (iv) aclimatación (adaptación fisiológica), y (v) selección de tolerancia heredada genéticamente (Medina et al. 2007).

En Chile, los efectos y decisiones relacionadas con el impacto de actividades productivas son regulados por la Ley 19,300, Bases Generales del Medio Ambiente y el Reglamento del Sistema de Evaluación de Impacto Ambiental (SEIA). Si bien este marco regulatorio ha funcionado para el control de la descarga de sustancias al ambiente, su aplicación considera normas secundarias aun no definidas que solo establecerán los valores de concentración y períodos máximos o mínimos permisibles de contaminantes, sin reflejar buena parte de los posibles efectos ecológicos derivados de la situación ambiental local (Reglamento, Artículo $6^{\circ}$ letra a, y Artículo $7^{\circ}$ ). Esta regulación considera también la definición de una serie de características del proyecto o actividad que, al ser subjetivas, dejan a criterio del titular del proyecto, de la opinión pública y/o de los evaluadores ambientales, el nivel de efectos que generará en los recursos naturales renovables (Artículo $6^{\circ}$, letras b-p). En otras palabras, la legislación vigente no considera la posible relación causal entre la intensidad, la duración y la frecuencia de una sustancia que es descargada al ambiente y los potenciales efectos a largo plazo que esta podría generar en la estructura, dinámica y funcionamiento de los ecosistemas expuestos (Medina \& Encina 2003).

Parte de este problema ha sido resuelto en países con mayor experiencia en temas ambientales, donde los posibles efectos son definidos basándose en la probabilidad de que daños ecológicos lleguen a expresarse dentro de valores de seguridad aceptables. Este concepto, denominado Evaluación de Riesgo Ambiental, ha sido desarrollado y utilizado en los últimos 20 años por distintas organizaciones y servicios con competencia ambiental en países de la Comunidad Europea y Estados Unidos (EC 1991, USEPA 1998). En esta línea, Europa modificó la gestión de la calidad de sus cuerpos de agua (i.e. European Water Framework Directive), pasando de objetivos de calidad basados exclusivamente en la química de estos (i.e. normas secundarias), hacia la evaluación tanto del estado químico como de la situación ecológica de estos sistemas naturales (EC 2002). Para lo anterior, la Comunidad Europea recientemente dicto el Reglamento No. 1907/2006 relativo al registro, evaluación, autorización y restricción de las sustancias y preparados químicos (REACH, de su sigla en inglés). El reglamento establece que para poder evaluar el riesgo ambiental asociado a la liberación de una sustancia al ambiente, los fabricantes e importadores deben obtener información sobre estos compuestos mediante la realización de los ensayos ecotoxicológicos alternativos que sean necesarios para estimar este riesgo (EC 2007).

Si bien en la legislación ambiental chilena y particularmente en algunas de las normas existentes se hace referencia a la probabilidad de daño ambiental a través de la palabra "riesgo", la manera en que este es considerado o cuantificado no ha sido aún regulada, estimándose de forma laxa y subjetiva. Una excepción a lo anterior está dada por el ya mencionado Instructivo para el Registro de Productos Farmacológicos elaborado por el 
SAG. Este instructivo complementa lo estipulado en el Reglamento de Productos Farmacéuticos de uso Exclusivamente Veterinario (Decreto Ministerio de Agricultura $\left.\mathrm{N}^{\circ} 25,2005\right)$ y en su Manual (P-PP-RM-002 2007), indicando los requisitos técnicos-legales que deben estar contenidos en los expedientes de solicitudes de registro normal (5 años) o provisional (1 año) de productos farmacológicos.

El registro de estos productos tiene por objeto verificar su calidad, eficacia e inocuidad para la salud humana, animal, vegetal y del medio ambiente en general. Para asegurar este último punto, en su sección $3 \mathrm{~A} 4$, el instructivo solicita la realización de una evaluación del riesgo ambiental asociado al uso del producto que se quiere registrar. Lo anterior implica desarrollar un estudio ecotoxicológico que permita estimar los efectos ambientales que podría provocar el uso del producto y definir las medidas que sean necesarias para reducir el riesgo (ver Apéndice).

El instructivo propone como guía para dar cumplimiento a este requisito las Fases I a IV de la metodología propuesta por The Veterinary International Cooperation on Harmonization (VICH). Esta guía de trabajo, aplicada en Japón, la Unión Europea y los Estados Unidos, indica la forma en que se debe realizar la evaluación de impacto ambiental de productos medicinales veterinarios (VMPs). A través de un árbol de decisión, la Fase I de la metodología VICH busca determinar si la liberación del VMP producirá algún daño ambiental, sobre la base del conocimiento disponible acerca de su eventual uso y forma de aplicación. Si las respuestas obtenidas en esta fase no son satisfactorias y entregan un marco inaceptable de incertidumbre se avanza a la Fase II, la cual busca generar información cuantitativa relacionada con el destino del producto en el ambiente una vez que es utilizado, y determinar su toxicidad aguda en especies no objetivo. La Fase II utiliza un sistema de etapas (Tiers) que sostienen el proceso de toma de decisión. La primera etapa (Tier A) recomienda utilizar técnicas ecotoxicológicas estándar (i.e. ISO, OECD) para caracterizar los potenciales efectos letales del producto en especies locales. Es importante mencionar aquí que si bien la guía VICH recomienda utilizar especies pertenecientes a tres "niveles taxonómicos", la utilización de este concepto es errado y debiera ser reemplazado por tres "especies pertenecientes a diferentes niveles tróficos".

Si los resultados de la primera etapa determinan que existe riesgo ambiental asociado al uso del producto ( $R Q>1$, ver Apéndice), se da paso a la segunda etapa (Tier B) en la cual se determinan los efectos subletales, en base a un diseño experimental estándar. Esta aproximación tiene por objetivo determinar la más alta concentración utilizada en la prueba de toxicidad subletal que no produce un efecto adverso en relación con el control (NOEC). Esta concentración es utilizada luego como PNEC en la evaluación de riesgo.

La inclusión de la metodología VICH en el sistema de registro de VMPs en Chile representa un avance significativo en las prácticas orientadas a la preservación del medio ambiente. Lo anterior por cuanto el desarrollo de las Fases I y II permite evaluar en forma objetiva los efectos del producto que se pretende utilizar, estableciendo un nivel de riesgo ambiental basado en la relación causal entre la concentración ambiental del VMP y sus potenciales efectos en diferentes especies locales ecológicamente relevantes. Sin embargo, la metodología VICH no es suficiente para reducir la incertidumbre con respecto a los posibles daños a largo plazo que el uso de un producto podría generar en la estructura, dinámica y funcionamiento de los ecosistemas expuestos. A continuación se analizan tres aspectos identificados en la metodología VICH estándar, que dificultarían la obtención de evidencia suficientemente confiable para el análisis de riesgo y la consecuente toma de decisión basada en su evaluación. Estos constituyen aspectos metodológicos deficitarios que, a nuestro juicio, pueden y deben ser reconsiderados en el corto plazo.

\section{Utilización de organismos y tiempos de expo- sición estándar}

Tanto en su Tier A como en su Tier B, la guía VICH propone el uso de normas ISO y de la OECD para el cálculo de PNEC bajo concentraciones letales y subletales. Para determinar el efecto de un producto veterinario sobre especies pertenecientes a diferentes niveles tróficos, estas normas presentan 
protocolos de trabajo donde las especies, su estado de desarrollo, el tiempo de duración de la exposición y los regímenes de esta son estándar y, por tanto, independientes del tipo de producto a evaluar y de su forma de aplicación.

La utilización de estos métodos estándar busca reducir la variabilidad de los resultados y permitir establecer comparaciones entre la sensibilidad de distintas especies o la toxicidad de diferentes sustancias (Stark \& Banken 1999). Sin embargo, las condiciones de experimentación que sugieren podrían distar significativamente de la forma en que el producto ingresará al ecosistema de interés, asî como de las condiciones naturales en las que se encuentran las poblaciones expuestas. Por ejemplo, la norma ISO 14669, sugerida por la metodología VICH en su Tier A, describe la utilización del copépodo Acartia tonsa (Copepoda: calanoidea) para la estimación del PNEC de una sustancia que será vertida a un ambiente costero. La norma indica que deben ser utilizados organismos adultos y que la exposición debe ser continua por un período de a lo menos $48 \mathrm{~h}$, para obtener así la concentración que mata a un $50 \%$ de las población expuesta en dicho período (LC 50 48 h). La Fig. 1 muestra el resultado de la aplicación de esta metodología en la evaluación de cipermetrina, piretroide sintético (plaguicida) utilizado en diferentes países para el control del Sea Lice en salmonicultura (Hart et al. 1997). La curva que se obtiene al graficar la mortalidad acumulada de los adultos en función de la concentración del plaguicida, arroja un LC 50 - $48 \mathrm{~h}$ de $0.33 \mu \mathrm{g} \mathrm{L}^{-1}$ (I.C. 95 $\%=0.28-0.43)$. Sin embargo, al repetir el experimento utilizando los estados tempranos de desarrollo (nauplii), la mortalidad en el mismo período de exposición aumenta significativamente, siendo los nauplii 24 veces más sensibles que los adultos (LC $50-48 \mathrm{~h}=$ 0.014 , I.C. $95 \%=0.012-0.018)$. Esta variación en la sensibilidad, dependiente del estado de desarrollo de los organismos expuestos, es una característica que ha sido descrita para numerosas especies de invertebrados utilizados en la evaluación de riesgo ambiental (Medina et al. 2002). Esta variación intrapoblacional en la sensibilidad puede ser considerablemente mayor que la variación observada entre especies diferentes (ver por ejemplo RamosJiliberto et al. 2004).

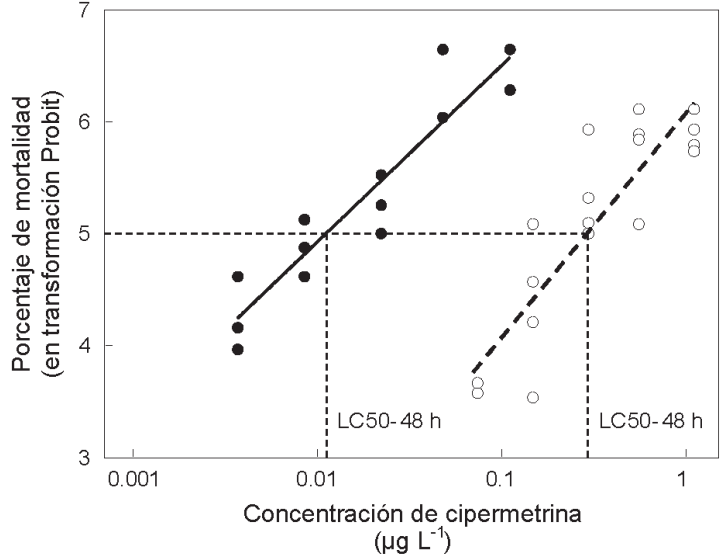

Fig. 1: Curvas concentración-mortalidad de $A$. tonsa nauplii (círculos negros) y adultos (círculos blancos), expuestos a cipermetrina. Datos de Medina et al. (2002).

Concentration-mortality curves of A. tonsa nauplii (closed circles) and adult (open circles) stages, exposed to cypermethrin. Data from Medina et al. (2002).

Los resultados que muestra la Fig. 1 sugieren por lo tanto, que la aplicación de la norma ISO 14669 en este caso es insuficiente, ya que no protege el estado de desarrollo más sensible de la especie utilizada. Así, la exposición a bajos niveles de cipermetrina por parte de una población natural, en la cual coexisten individuos en diferentes fases de su desarrollo, afectaría la sobrevivencia y desarrollo de los individuos más jóvenes, lo que puede traducirse en una perturbación del crecimiento, abundancia y/o estructura poblacional de la especie evaluada.

A nivel intrapoblacional, la estimación de los efectos de la mortalidad diferencial por edades $-\mathrm{o}$ estados del ciclo de vida- puede abordarse a través de métodos de modelación matricial del ciclo de vida y sus correspondientes análisis de perturbación prospectivo y retrospectivo (Caswell 2001), a fin de detectar los estados más sensibles a la perturbación y sus consecuencias en la tasa de crecimiento poblacional y en la estructura de estados esperada a largo plazo (Ramos-Jiliberto \& Aránguiz-Acuña 2007). Dado que las interacciones tróficas en particular, y las ecológicas en general, a menudo varían a través de la ontogenia, cambios en las abundancias relativas de cada estado conllevan cambios en la magnitud de las interacciones entre la 
especie estudiada y el resto de la comunidad (Werner \& Gilliam 1984, Stark \& Banken 1999). Las alteraciones en la magnitud de las interacciones interespecíficas directas pueden ejercer un impacto en la estructura, dinámica y persistencia de la comunidad completa (Kondoh 2003).

Como se señaló, las normas indicadas en la metodología VICH también estandarizan los tiempos de duración de las evaluaciones. Del ejemplo anterior, es posible predecir la ocurrencia de mortalidades significativas en los copépodos expuestos a cipermetrina por más de $48 \mathrm{~h}$ a concentraciones dos órdenes de magnitud menores que la utilizada en el tratamiento del Sea Lice. Bajo estas condiciones, la concentración ambiental esperada de cipermetrina (PEC) será mayor que la concentración sin efecto (PNEC) y por tanto, existirá un riesgo ambiental asociado al uso de este plaguicida para el control de este parásito en salmones. Sin embargo, la aplicación de este método no simula el régimen de exposición al que estarían sometidos los organismos no objetivo expuestos al contacto con este plaguicida. Lo anterior, por cuanto el tratamiento que utiliza este producto solo dura 30-60 min, luego del cual la cipermetrina es liberada al ambiente sin poder ser detectada al cabo de $2 \mathrm{~h}$ postratamiento (Hunter \& Fraser 1995). En consecuencia, la metodología estándar indicada en la norma ISO 14669 no considera una representación adecuada de las condiciones de exposición reales, eliminando del análisis la posible existencia de una toxicidad retrasada o de una recuperación de los organismos expuestos. La Fig. 2 muestra un ejemplo de lo anterior. Las curvas indican la sobrevivencia de A. tonsa (adultos) después de $1 \mathrm{~h}$ de exposición a cipermetrina en diferentes concentraciones. Si bien se observan efectos en la motilidad de los organismos expuestos durante el periodo de exposición, la mortalidad de estos no superó el $10 \%$ (Medina et al. 2004). Durante los siete días que siguieron al período de exposición, la sobrevivencia de las poblaciones expuestas se redujo significativamente, demostrando la existencia de toxicidad retrasada luego de una exposición corta a cipermetrina. Sin embargo, la mortalidad observada durante el período postexposición no superó el $50 \%$ en ninguno de los tratamientos evaluados (i.e. < LC 50 - 144 h).

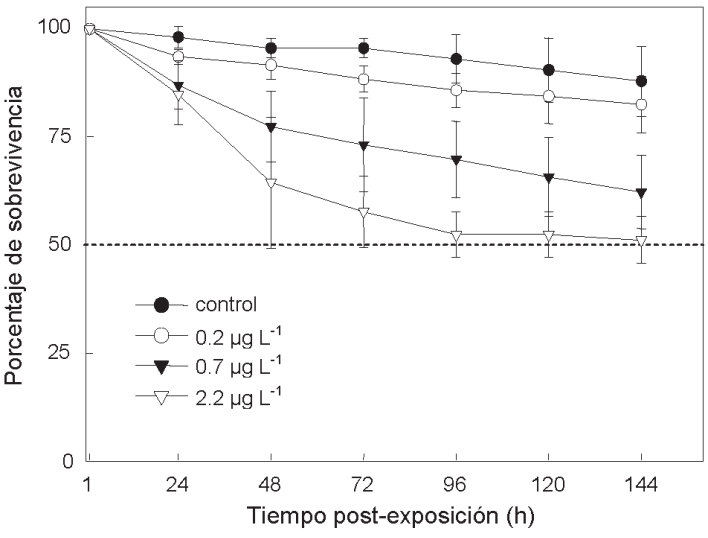

Fig. 2: Sobrevivencia de A. tonsa luego de $1 \mathrm{~h}$ de exposición a cipermetrina. Se indica la media \pm DE. Datos de Medina et al. (2004).

Survival of A. tonsa after $1 \mathrm{~h}$ exposure to cypermethrin. Mean \pm SD are shown. Data from Medina et al. (2004).

En la literatura científica existen dos puntos de vista en relación al uso de ensayos de toxicidad con una duración estándar. Por un lado, algunos autores sugieren que estas metodologías sobreestiman los potenciales impactos ya que en exposiciones reales, las sustancias utilizadas no persisten en el ambiente por periodos comparables (Clark et al. 1987). Por el contrario, el uso de metodologías estándar estaría subestimando las posibles consecuencias derivadas de exposiciones prolongadas o como resultado de una toxicidad retrasada, ya que solo miden la mortalidad durante el periodo de exposición experimental (Jensen \& Forbes 2001). Si bien los resultados indicados en la Fig. 2 dan cuenta de una toxicidad retrasada asociada a exposiciones cortas de cipermetrina, en general apoyan la primera de estas dos posiciones por cuanto la mortalidad asociada a una exposición más cercana a la realidad $(1 \mathrm{~h})$ después de un tratamiento contra Sea Lice, sería menor que la observada durante el desarrollo de la evaluación estándar propuesta por la guía VICH.

De lo anterior es posible concluir que si bien los métodos estándar sugeridos por la metodología VICH permiten la comparación de sensibilidades entre distintas especies y también la comparación de toxicidad entre diferentes sustancias, estos no necesariamente representan escenarios adecuados para evaluaciones del riesgo ambiental asociado al uso de VMPs en acuicultura. 


\section{Diseño experimental y análisis estadístico}

Si los resultados del Tier A determinan que existe riesgo ambiental asociado al uso del producto $(\mathrm{RQ}>1)$, la guía VICH propone la aplicación de un diseño basado en una prueba de hipótesis para estimar los efectos subletales que podría generar el producto (guía VICH, Tier B). En este caso, los parámetros estudiados corresponden a aquellos efectos crónicos sobre caracteres demográficos (e.g. desarrollo, crecimiento, reproducción) o fisiológicos (e.g. ingestión, respiración) por lo general evaluados durante exposiciones mayores a 96 h. Esta metodología busca determinar la máxima concentración que no produce efecto observable (NOEC) y aquella que produce el menor efecto observable (LOEC), mediante la comparación de las respuestas obtenidas bajo diferentes concentraciones del producto con respecto a un grupo control. La hipótesis nula considera que la respuesta bajo las diferentes concentraciones del producto estudiado es igual a la respuesta observada en el grupo control. Si la hipótesis es rechazada, la concentración más alta bajo la cual no se observa un efecto significativo es la NOEC y la más baja en la cual se produce el efecto es la LOEC. En esta aproximación de riesgo ambiental las concentraciones a las cuales los organismos son expuestos responden a una escala geométrica arbitrariamente escogida por el investigador. Las comparaciones entre las respuestas obtenidas se realizan mediante diferentes métodos estadísticos, dentro de los cuales destacan el Análisis de Varianza y los test a posteriori Tukey y Dunnett (Zar 1999).

La Fig. 3 muestra el resultado de la aplicación de un Tier B en que se determinó la tasa de ingestión de $A$. tonsa bajo diferentes concentraciones de cipermetrina. Este microcrustáceo, que abunda en gran parte de las costas del mundo (Tester 1985), contribuye al traspaso de la energía desde organismos autótrofos a niveles superiores de la trama trófica a través de interacciones depredadorpresa. Por esta razón, cualquier efecto en su tasa de ingestión podría escalar hacia una alteración de la dinámica y el funcionamiento del ecosistema estudiado. La Fig. 3 indica que hasta concentraciones de $0.023 \mu \mathrm{g} \mathrm{L}^{-1}$, la cipermetrina no produce un efecto significativo
(NOEC). Por el contrario, a concentraciones iguales o mayores a $0.072 \mu \mathrm{g} \mathrm{L}^{-1}$ el plaguicida produce un efecto negativo significativo (LOEC).

Si bien el resultado entregado por la prueba estadística utilizada es en este caso útil para conocer ciertos niveles de concentración a los que se produce un efecto, el valor de NOEC no necesariamente se relaciona con la ocurrencia de efectos con relevancia ecológica observables a niveles iguales e incluso inferiores a este umbral. Lo anterior, dado que el número de tratamientos y las concentraciones específicas utilizadas en el experimento han sido arbitrariamente escogidas por el investigador. Así, el tipo de análisis estadístico aplicado en la metodología VICH resta precisión y peso a la evidencia que se entrega en la evaluación de riesgo ambiental de un producto veterinario.

Una mayor precisión y significancia ecológica en un estudio de este tipo puede lograrse mediante la aplicación de análisis regresionales. Moore \& Caux (1997) concuerdan en que este tipo de análisis produce más y mejor información para ser utilizada en la evaluación de riesgo ambiental y para la generación de criterios de calidad ambiental. La Fig. 4 muestra los mismos resultados de la Fig. 3 , esta vez analizados utilizando la aproximación regresional. Los datos han sido

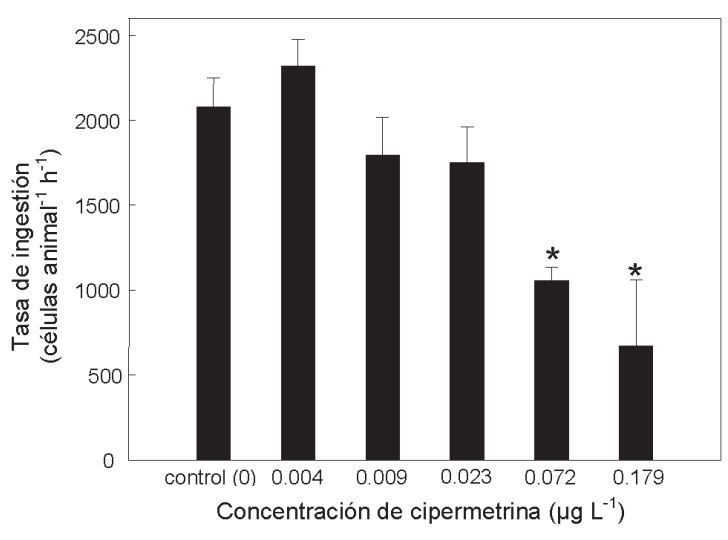

Fig. 3: Tasa de ingestión de A. tonsa expuesta a diferentes concentraciones de cipermetrina. Los asteriscos indican diferencias significativas (ANOVA $\mathrm{F}_{5,23}=31.07 ; \mathrm{P}<0.001$ ) con respecto al control. Se indica la media $\pm \mathrm{DE}$.

Ingestion rate of $A$. tonsa exposed to several concentrations of cypermethrin. Asterisks indicate significant differences $\left(\right.$ ANOVA $\left.F_{5,23}=31.07 ; \mathrm{P}<0.001\right)$ from control. Mean $\pm \mathrm{SD}$ are shown. 
ajustados a un modelo sigmoideo, con el cual las concentraciones que producen efectos específicos de interés ecológico pueden ser determinadas con precisión. En el ejemplo aquí utilizado, las concentraciones que producen un $30 \%$ y un $50 \%$ de inhibición corresponden respectivamente a $0.038 \mu \mathrm{g} \mathrm{L}^{-1}$ y $0.083 \mu \mathrm{g} \mathrm{L}^{-1}$.

\section{Indiferencia respecto a efectos supraindivi- duales}

Los efectos tanto letales como subletales de contaminantes medidos a través de pruebas de toxicidad aguda y crónicas respectivamente, evalúan la respuesta de poblaciones $u$ organismos individuales aislados a la exposición de los compuestos de interés. Sin embargo, efectos relevantes a nivel comunitario escapan al análisis que solicita el instructivo. Así por ejemplo, concentraciones de productos por debajo de niveles detectados de LOEC y NOEC podrían ejercer alteraciones en la capacidad de respuesta de los organismos a sus depredadores. En estos casos, la presencia del producto podría inhibir el desarrollo de respuestas defensivas en las presas, con el consecuente aumento en mortalidad por

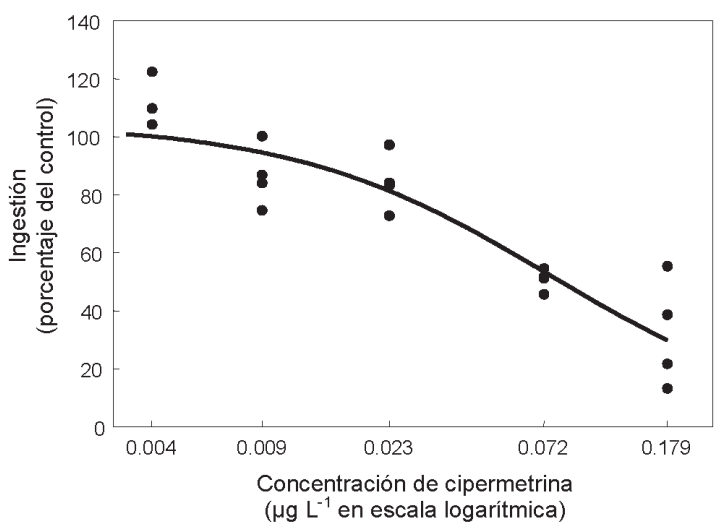

Fig. 4: Tasa de ingestión relativa de A. tonsa como función de la concentración de cipermetrina. Se muestran los mismos datos utilizados para la Fig. 3, pero estos se muestran por réplica (círculos negros) y como distancias respecto del control. La línea continua muestra un modelo logístico ajustado a estos datos $\left(r^{2}=0.84\right)$.

Relative ingestion rate of A. tonsa as a function of cypermethrin concentration. Same data for Fig. 3 is used here; however, these are depicted as replicates and in terms of distances from the control. The continuous line indicates a logistic model fitted to the data $\left(r^{2}=0.84\right)$. depredación. En otros, el producto podría emular las señales de los depredadores y estimular innecesariamente el desarrollo de defensas, con el consiguiente costo fisiológico o ecológico asociado (ver Lürning \& Sheffer 2007). La Fig. 5 presenta gráficamente estos procesos, donde la especie presa (en rectángulo) puede presentar un estado normal no defendido ( $\mathrm{x}$ ) o un estado defendido ( $\left.\mathrm{x}^{\prime}\right)$. El paso al estado defendido en la presa es gatillado por la percepción de señales (k) liberadas por el depredador (y) e impone ciertos costos que se traducen en una pérdida de biomasa en la población (flechas inferiores). En una situación prístina (sin contaminantes), una población de presas en ausencia de depredadores crece a una tasa que incluye pérdidas naturales por metabolismo o muerte. En presencia de riesgo de depredación, la presa transita a un estado defendido ( $x^{\prime}$ ) que minimiza las muertes por depredación pero con el costo poblacional antes mencionado (representado por la flecha gruesa inferior). Un producto (s) que es vertido al ambiente puede actuar induciendo el tránsito de la presa al estado defendido en ausencia de depredación, generando el costo poblacional sin el beneficio activo de la protección; o bien interfiriendo en la percepción de las señales liberadas por el depredador, con lo que se impide el paso al estado defendido y con ello la reducción de las pérdidas por depredación.

En este escenario, el uso de técnicas ecotoxicológicas estándar y la medición de respuestas en individuos o poblaciones aisladas son de poca o nula utilidad para el reconocimiento de potenciales efectos nocivos de ciertas sustancias sobre las comunidades naturales. En lenguaje ecológico, este tipo de alteración subletal expresada solo a nivel comunitario se denominan modificación de interacciones o efectos indirectos mediados por rasgos (Wootton 1994, Abrams et al. 1996). Estos se traducen en una alteración de la arquitectura de interacciones entre las poblaciones que componen una comunidad.

Por otro lado, tanto los efectos letales como los subletales que los contaminantes ejercen sobre una población determinada tienen el potencial de propagarse a través de toda la red de interacciones ecológicas, con consecuencias difícilmente predecibles a partir de la información obtenida desde ensayos de 
Sin depredador

Con depredador

(A)

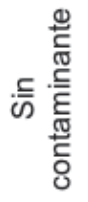

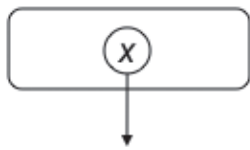

(C)

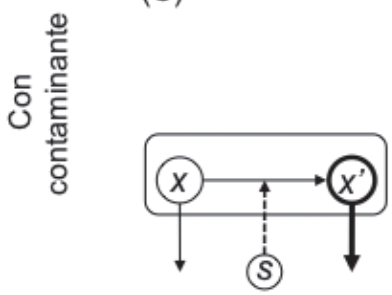

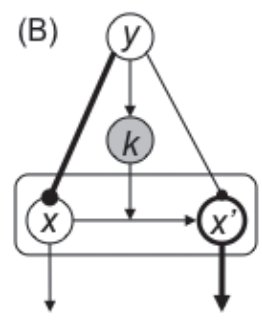

(D)

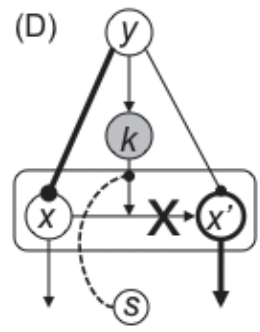

Fig. 5: Alteración mediada por contaminantes de la respuesta poblacional al riesgo de depredación. A: en ausencia de depredadores y contaminantes la tasa de crecimiento de la población $x$ es reducida solo por gastos de mantenimiento (flechas inferiores descendentes). B: en presencia de depredadores (y) la población x percibe la señal de riesgo ( $\mathrm{k}$, en gris) $y$ transita a un estado defendido $x$ ', en el cual se reduce la depredación al costo de incrementar el gasto. Un contaminante (s) puede emular la señal de depredación con el consiguiente aumento del gasto de mantenimiento de X' (C), o inhibir (indicado por X) la transición al estado defendido en presencia de depredadores con el consiguiente aumento de la mortalidad por depredación (D). Las flechas terminadas en punta y en círculo representan efectos positivos y negativos respectivamente. El espesor de las flechas indica su magnitud relativa.

Stressor-mediated alteration of the population response to predation risk. A: in the absence of predators or stressors, the growth rate of population $\mathrm{x}$ is reduced only by the maintenance expenditure (bottom downward arrows). B: in presence of predators (y) population $\mathrm{x}$ perceives the risk signal $(\mathrm{k}$, in grey) and transits to a defended state $\mathrm{x}$ ', in which predation is reduced at a cost of an increased expenditure. A stressor (s) can emulate the predation signal with the subsequent increase in maintenance expenditure of $\mathrm{x}$ ' (C), or inhibit (shown by $\mathrm{X}$ ) the transition to defended state in presence of predators with the subsequent increase in predation-driven mortality (D). Links ending in arrowheads and circles represent positive and negative effects, respectively. Relative strength is indicated by the thickness of links.

especies aisladas. La transmisión de los efectos derivados de la exposición a contaminantes se produce por una cadena de interacciones directas entre especies físicamente vinculadas (Wootton 1994), fenómeno también llamado efecto indirecto mediado por densidad (Abrams et al. 1996) (Fig. 6A), o bien a través de la alteración de la magnitud de interacción entre pares de especies directamente conectadas (Fig. $6 \mathrm{~B})$. El potencial de propagación de un efecto nocivo generado por contaminantes depende de atributos globales tales como el número de especies interactuantes, la densidad de interacciones, y los patrones específicos de conexiones entre las especies componentes de la comunidad (ver Pascual \& Dunne 2006).

Existen dos aproximaciones teóricas que aparecen adecuadas para la representación y estudio de este tipo de efectos complejos en ensambles multivariados. La primera corresponde a la modelización cualitativa de sistemas complejos basada en el análisis de la matriz comunitaria, también llamada análisis de ciclos o loop analysis (ver Puccia \& Levins 1985, Dambacher \& Ramos-Jiliberto 2007). Esta aproximación permite evaluar la
(A)

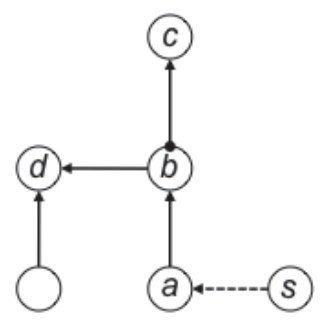

(B)

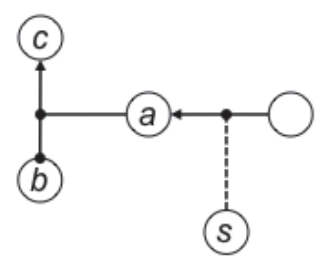

Fig. 6: Propagación dentro de la comunidad de los efectos de un contaminante (s). A: efectos indirectos mediados por densidad, propagándose por las especies a, b, c y d. B: efectos indirectos mediados por rasgos, propagándose por las especies a, b y c. Las flechas terminadas en punta y en círculo representan efectos positivos y negativos respectivamente. Nótese que entre las especies b y c existe una interacción negativa/positiva, tipo depredador-presa.

Propagation within a community of the effects of a stressor (s). A: density-mediated indirect effects propagating through species a, b, c, and d. B: trait-mediated indirect effects propagating through species $a, b$, and c. Links ending in arrowheads and circles represent positive and negative effects, respectively. Note that between species $b$ and $\mathrm{c}$ there exists a negative/positive interaction of the type predator-prey. 
estabilidad local de sistemas ecológicos multiespecíficos y los cambios en densidad de equilibrio de las poblaciones componentes como respuesta a alteraciones en el desempeño local de una o varias poblaciones primariamente afectadas por un estresante. La segunda, es la aproximación de sistemas complejos derivada de la mecánica estadística (Albert \& Barabási 2002, Newman 2003, Pascual \& Dunne 2006), que permite establecer asociaciones entre la arquitectura de grandes ensambles y sus propiedades dinámicas globales, tales como la fragilidad y robustez de la red ecológica completa a perturbaciones específicas como la extinción local de especies (Valdovinos et al. 2009, Ramos-Jiliberto et al. 2009).

\section{CONCLUSIONES Y PROPUESTAS}

La acuicultura se ha transformado en un importante componente de la economía nacional y su aporte será significativo en el proceso de convertir a Chile en una potencia alimentaria. Sin embargo, la estrecha relación que posee con el ambiente genera conflictos que son hoy de gran interés público. La aplicación de la metodología VICH indicada en el Instructivo Técnico para el Registro de Productos Farmacológicos elaborado por el SAG representa un importante avance hacia el control de los impactos que podría generar la liberación de VMP utilizados para el control de parásitos y otras enfermedades que afectan a los organismos en cultivo. Los métodos propuestos por esta metodología permiten obtener información sobre los niveles de exposición que produce cambios en la mortalidad, crecimiento, reproducción y fisiología de los organismos no objetivo que son expuestos. Sin embargo, este tipo de ensayos no reflejan lo que ocurre luego de una administración real de VMP, no permiten relacionar los efectos observados con alteraciones demográficas naturales y no entregan información sobre los efectos indirectos que podrían ocurrir en ecosistemas multiespecíficos complejos.

El objetivo principal de la evaluación de riesgo ambiental es cuantificar la probabilidad que el producto estudiado produzca un efecto adverso en poblaciones y comunidades ecológicas (Forbes et al. 2008). Lo anterior dado que es a este nivel de organización natural que el efecto sobre los servicios y funciones que entrega la naturaleza es relevante. Por un lado, buena parte de las disminuciones poblacionales severas son generadas por cadenas de efectos que involucran a un conjunto amplio de especies (Griffen \& Drake 2008), y por otro, es solo a este nivel que la sociedad en general puede percibir las variaciones producidas por el desarrollo de actividades productivas. En otras palabras, aun cuando no se altere directamente a especies carismáticas o económicamente importantes (i.e. cisnes, choritos, etc.), los efectos de una actividad productiva generarán preocupación social a posteriori si se asocian a una variación observable de la abundancia de poblaciones locales naturales.

Considerando que la relación entre los efectos individuales y la dinámica de poblaciones y comunidades expuestas es por defecto compleja y no lineal (Ferson et al. 1996), la aplicación de un mayor esfuerzo en el desarrollo de una aproximación mixta, en que se ejecuten experimentos controlados a mayor escala que la usual (más especies, mayor volumen, tiempos más largos) junto a la modelización de sistemas ecológicos, surge como una mejor alternativa para la correcta extrapolación de los potenciales impactos asociados a la utilización de VMP en acuicultura. En este contexto, la experimentación con mesocosmos aparece como una posibilidad eficiente, que combina una escala aceptable de trabajo con la posibilidad de control experimental y replicación. Como se describe en la Fig. 7, el trabajo con micro y mesocosmos permitiría la observación de los efectos directos e indirectos que la utilización del producto produce en las comunidades expuestas, así como una estimación de las posibilidades de recuperación que tendrían los sistemas ecológicos naturales (Medina et al. 2004).

En EE.UU. y países de la comunidad Europea, la utilización de mesocosmos ha sido propuesta como una metodología orientada a confirmar y ampliar la información surgida de los estudios que se efectúan en laboratorio (SETAC 1991; SETAC-RESOLVE 1992). Sin embargo, en el contexto del Instructivo Técnico para el Registro de Productos Farmacológicos, la aplicación primaria de este tipo de estudios entregaría evidencia suficientemente confiable 


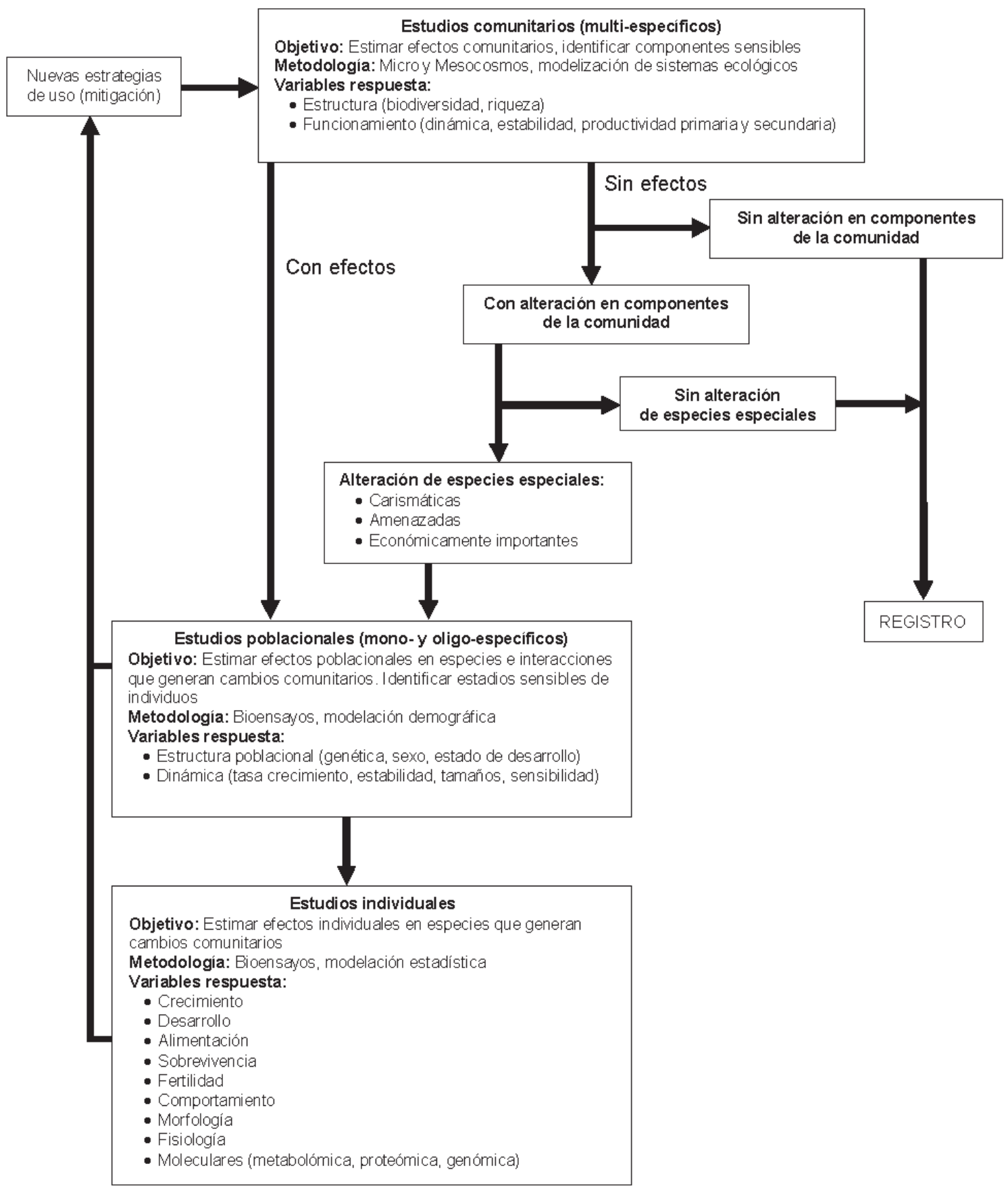

Fig. 7: Nueva propuesta, en forma de árbol de decisiones, para la estimación de riesgo ambiental de VMPs utilizados en acuicultura.

A new proposal, in form of a decision tree, for the ecological risk assessment of VMPs used in aquaculture. 
para el análisis de riesgo y la consecuente toma de decisión basada en su evaluación. La determinación de efectos en la estructura y funcionamiento de los mesocosmos utilizados permitiría una decisión informada en relación al registro, que consideraría las respuestas que preocupan a los especialistas y a la sociedad en general. Una vez conocidos los efectos y estimado el riesgo a nivel comunitario mediante el uso de mesocosmos, el estudio podría (de ser necesario) proseguir mediante la aplicación de la metodología VICH en forma inversa. Si en la primera etapa se detectan efectos adversos a nivel comunitario, proponemos derivar hacia la indagación de los mecanismos poblacionales que generan los efectos comunitarios observados. De este modo, se posibilita el diseño de estrategias científicamente fundadas para la mitigación y/o rediseño de procedimientos para la correcta utilización del VMP. En este caso, los resultados obtenidos en el mesocosmos deberán ser analizados para identificar los componentes del sistema (poblaciones e interacciones) responsables de las variaciones estructurales o funcionales observadas en la comunidad. En esta etapa, el efecto del producto sobre la estructura y dinámica de las poblaciones sensibles identificadas deberá ser determinando mediante el desarrollo de bioensayos en laboratorio que consideren diferentes fases del ciclo de vida de los organismos, apoyado por métodos de modelación y análisis demográficos. Así, se podrá identificar los mecanismos de nivel jerárquico inferior responsables de las alteraciones a nivel comunitario. Con ello, se establece el tipo de organismo (e.g. edad, sexo) y los procesos individuales (e.g. fecundidad, desarrollo, metabolismo) más sensibles al VMP específico.

Esta etapa podrá continuar con el análisis de los efectos que el VMP produce a nivel individual, considerando las observaciones a la metodología VICH efectuadas en este documento (i.e. utilización de organismos y tiempos adecuados; utilización de diseños regresionales). La medición de respuestas a este nivel entregaría mayor información sobre las modificaciones que se deben efectuar a las estrategias de uso del VMP para evitar efectos ambientales significativos y lograr así su registro (ver Fig. 7).

Por otro lado, si la evaluación del VMP con mesocosmos no arroja efectos en la estructura y/o funcionamiento de la comunidad estudiada, los datos deben ser analizados con el objeto de descartar posibles alteraciones en los componentes de la comunidad. Si el análisis arroja variaciones en la abundancia o estructura de especies carismáticas, amenazadas o económicamente importantes, la misma evaluación poblacional e individual descrita anteriormente deberá ser aplicada con la intención de buscar medidas de mitigación o nuevas estrategias de aplicación.

Si bien diferentes tipos de mesocosmos han sido descritos en la literatura científica con el objeto de evaluar el riesgo ambiental asociado a productos liberados al ambiente (ver Medina et al. 2004), la mayoría corresponde a ambientes dulceacuícolas, siendo escaso el desarrollo de mescosmos marinos (Kuiper 1977, Medina et al. 2004). En los últimos años, Chile ha avanzado en forma significativa en el conocimiento oceanográfico y limnológico de sus cuerpos de agua y cuenta con grupos de investigadores altamente competentes teórica y metodológicamente en el ámbito de la ecología científica. El nivel alcanzado permitiría desarrollar este tipo de estudios multiespecíficos con una sólida base técnicocientífica. Sin embargo, la heterogeneidad de los ecosistemas acuáticos (costero, fiordos, canales, ríos, lagos) y la escasa experiencia existente sobre este tipo de trabajos en el mundo y en Chile en particular, obligan a invertir recursos y tiempo en el desarrollo de procedimientos fiables que aseguren la replicabilidad de los resultados y permitan así, aprovechar todo el potencial que esta metodología tendría en su aplicación. Mediante el desarrollo de una metodología general, que sea aceptada por todas las partes involucradas (i.e. autoridad, científicos, empresa, sociedad), la evaluación de riesgo ambiental utilizando esta aproximación empírico-teórica permitirá obtener la evidencia necesaria para revelar la posible ocurrencia de efectos causantes de cambios en la estructura, dinámica y funcionamiento de los ecosistemas expuestos.

\section{AGRADECIMIENTOS}

Los autores agradecen las sugerencias efectuadas por dos revisores anónimos, las que mejoraron el artículo significativamente. R.R.- 
J. agradece financiamiento parcial de proyecto FONDECYT 1090132.

\section{LITERATURA CITADA}

ABRAMS PA, BA MENGE, GG MITTELBACH, D SPILLER \& P YODZIS (1996) The role of indirect effects in food webs. En: Polis GA \& KO Winemiller (eds) Food webs. Integration of patterns and dynamics: 371-395. Chapman and Hall, York, Estados Unidos.

BARRIONUEVO A (2008) Salmon virus indicts Chile's fishing methods. The New York Times, 27 de Marzo. Nueva York, Estados Unidos. URL: http:// www.nytimes.com/2008/03/27/world/americas/ 27 salmon.html (accedido Junio 26, 2009).

BRAVO S, S SEVATDAL \& TE HORSBERG (2008) Sensitivity assessment of Caligus rogercresseyi to emmamectin benzoate in Chile. Aquaculture 282: 7 12.

BURRIDGE L, J WEIS, F CABELLO \& J PIZARRO (2008) Chemical use in salmon aquaculture: a review of current practices and possible environmental effects. Report of the Technical Working Group on Chemical use in salmon aquaculture of the World Wildlife Fund Salmon Aquaculture Dialogue. World Wildlife Fund, Washington, Estados Unidos. URL: http:// www.worldwildlife.org/what/globalmarkets/ aquaculture/WWFBinaryitem8842.pdf (accedido Junio 26, 2009).

BUSCHMANN A \& A FORTT (2005) Efectos ambientales de la acuicultura intensiva y alternativas para un desarrollo sustentable. Revista Ambiente y Desarrollo (Chile) 21: 58-64.

BUSCHMANN A, V RIQUELME, M HERNÁNDEZGONZÁLEZ, D VARELA, JE JIMÉNEZ et al. (2006) A review of the impacts of salmonid farming on marine coastal ecosystem in the southeast Pacific. ICES Journal of Marine Science 63: 13381345 .

CABELLO FC (2004) Antibióticos y acuicultura en Chile: consecuencias para la salud humana y animal. Revista Médica de Chile 132: 1001-1006.

CASWELL H (2001) Matrix population models: Construction, analysis, and interpretation. Second edition. Sinauer Associates, Estados Unidos. 722 $\mathrm{pp}$.

CLARK J, P BORTHWICK, L GOODMAN, J PATRICK, E LORES \& J MOORE (1987) Comparison of laboratory toxicity test results with responses of estuarine animals exposed to fenthion in the field. Environmental Toxicology and Chemistry 6: 151160.

CNIC (2007) Hacia una estrategia nacional de innovación para la competitividad. Vol.1. Consejo Nacional de Innovación para la Competitividad. Gobierno de Chile. Santiago, Chile. 189 pp. URL: http:// www.cnic.cl/content/view/468181/Hacia-unaEstrategia.html (accedido Junio 26, 2009).

COSTA-PIERCE BA, A BUSCHMANN, S CROSS, JL IRIARTE, Y OLSEN \& G REID (2008) Nutrient impacts of farmed Atlantic salmon (Salmo salar) on pelagic ecosystems and implications for carrying capacity. Report of the Technical Working Group on Nutrients and Carrying Capacity of the World Wildlife Fund Salmon Aquaculture Dialogue.
World Wildlife Fund, Washington, Estados Unidos. URL: http://www.worldwildlife.org/cci/pubs/ Nutrient_Impacts_SalmonCulture_Commissioned_ By_Salmon_Dial.pdf (accedido Junio 26, 2009).

DAMBACHER JM \& R RAMOS-JILIBERTO (2007) Understanding and predicting effects of modified interactions through a qualitative analysis of community structure. The Quarterly Review of Biology 82: 227-250

EC (1991) Guide document on Aquatic Ecotoxicology. Sanco/3268/2001 ver 4. Directive 414. European Commission Health and Consumer Protection. Directorate General. Bruselas, Bélgica. $62 \mathrm{pp}$.

EC (2002) Establece un marco comunitario de actuación en el ámbito de la política de aguas. Directive 60 . European Commission. Directorate General. Bruselas, Bélgica. 72 pp.

EC (2007) Reglamento (CE) n 1907/2006 del Parlamento Europeo y del Consejo de 18 de diciembre de 2006 relativo al registro, la evaluación, la autorización y la restricción de sustancias y preparados químicos $(\mathrm{REACH})$. European Commission. Directorate General. Bruselas, Bélgica. 852 pp.

FERSON S, LR GINZBURG \& RA GOLDSTEIN (1996) Inferring ecological risk from toxicity bioassays. Water Air and Soil Pollution 90: 71-82.

FOLKE C, N KAUTSKY, H BERG, A JANSSON \& M TROELL (1998) The ecological footprint concept for sustainable seafood production: a review. Ecological Applications 8: 63-71.

FORBES V, P CALOW \& R SIBLY (2008) The extrapolation problem and how population modeling can help. Environmental Toxicology and Chemistry 27: 1987-1994.

GOLDBURG R \& R NAYLOR (2005). Future seascapes, fishing, and fish farming. Frontier in Ecology and the Environment 3: 21-28

GOWEN RJ \& NB BRADBURY (1987) The ecological impact of salmonid farming in coastal waters: A review. Oceanography and Marine Biology: an Annual Review 25: 563-575.

GRIFFEN BD \& JM DRAKE (2008) A review of extinction in experimental populations. Journal of Animal Ecology 77: 1274-1287.

HALLAEGRAEFF GM (1993) A review of harmful algal blooms and their apparent global increase. Phycologia 32: 79-99.

HART J, J THACKER, J BRAIDWOOD, N FRASER \& J MATTHEWS (1997) Novel cypermethrin formulation for the control of sea lice on salmon (Salmo salar). The Veterinary Records 140: 179181.

HUNTER R \& N FRASER (1995) Field monitoring of the effects of cypermethrin as GPRD01. Ref. GP95033. Brampton: Grampian pharmaceuticals limited. Research division. 76 pp.

JENSEN A \& V FORBES (2001) Interclonal variation in the acute and delayed toxicity of cadmium to the European prosobranch gastropod Potamopyrgus antipodarum (Gray). Archives of Environmental Contamination and Toxicology 40: 230-235.

KONDOH M (2003) Foraging adaptation and the relationship between food-web complexity and stability. Science 299: 1388-1391.

KUIPER J (1977) Development of North sea coastal plankton communities in separate plastic bags under identical conditions. Marine Biology 44: 97-107.

LEÓN-MUÑOZ J, D TECKLIN, A FARÍAS \& S DÍAZ (2007) Salmonicultura en los lagos del sur de Chile Ecorregión Valdiviana. Report of the Technical 
Working Group on Salmon Farming in lakes of the World Wildlife Fund Salmon Aquaculture Dialogue. World Wildlife Fund, Washington, Estados Unidos. URL: http://www.wwf.cl/ archivos_publicaciones/informe $\% 20$ salmones \%20lagos\%20sur\%20de\%20Chilerestriccion.pdf (accedido Junio 26, 2009).

LÜRNING M \& M SCHEFFER (2007) Info-disruption: Pollution and the transfer of chemical information between organisms. Trends in Ecology and Evolution 22: 374-379.

MEDINA M, C BARATA, T TELFER \& DJ BAIRD (2002) Age-and sex related variation in sensitivity to the pyrehtroid cypermethrin in the marine copepod Acartia tonsa Dana. Archives of Environmental Contamination and Toxicology 42: 17-22.

MEDINA M \& F ENCINA (2003) Incorporación de la Evaluación de Riesgo Ecológico en el Sistema de Evaluación de Impacto Ambiental para ecosistemas acuáticos en Chile. Revista Ambiente y Desarrollo (Chile) 19: 19-26.

MEDINA M, C BARATA, T TELFER \& DJ BAIRD (2004) Effects of cypermethrin on marine plankton communities. A simulated field study using mesocosms. Ecotoxicology and Environmental Safety 58: 236-245.

MEDINA MH, JA CORREA \& C BARATA (2007) Microevolution due to pollution: Possible consequences for ecosystem responses to toxic stress. Chemosphere 67: 2105-2114.

MOORE D \& P CAUX (1997) Estimating the low toxic effects. Environmental Toxicology of Chemistry 16: 794-801.

NORTON S, RODIER D, J GENTILE, M TROYER, R LANDY \& W VAN DER SCHAILE (1995) The EPA's Framework for Ecological Risk Assessment. En: Hoffman DJ, BA Ratter, GA Burton \& J Cairns (eds) Handbook of Ecotoxicology: 703-716. Lewis Publishers, New York, Estados Unidos.

PARKER ED, VE FORBES, SL NIELSEN, C RITTER, C BARATA et al. (1999) Stress in ecological systems. Oikos 86: 179-184.

PASCUAL M \& JA DUNNE (2006) Ecological networks: linking structure to dynamics in food webs. Oxford University Press, New York, Estados Unidos. 386 pp.

PUCCIA CJ \& R LEVINS (1985) Qualitative modeling of complex systems. An introduction to loop analysis and time averaging. Harvard University Press, Cambridge, MA, Estados Unidos. 259 pp

RAMOS-JILIBERTO R, P DAUELSBERG \& LR ZÚÑIGA (2004) Differential tolerance to ultraviolet-B light and photoenzimatic repair in cladocerans from a Chilean lake. Marine and Freshwater Research 55: 193-200.

RAMOS-JILIBERTO R \& A ARÁNGUIZ-ACUÑA (2007) Between-species differences in demographic responses to temperature of coexisting cladocerans. Austral Ecology 32: 766-774.

RAMOS-JILIBERTO R, AA ALBORNOZ, FS
VALDOVINOS, C SMITH-RAMIREZ, M ARIM, JJ ARMESTO \& PA MARQUET (2009) A network analysis of plant-pollinator interactions in temperate rain forests of Chiloé Island, Chile. Oecologia 160: 697-706.

REEL M (2007) Chile's flourishing fish farms prompt fears for ecosystems. The Washington Post, 2 de Diciembre. Washington, Estados Unidos. URL: http://www.washingtonpost.com/wp-dyn/content/ article/2007/12/01/AR2007120100763.html (accedido Junio 26, 2009).

SETAC (1991) Guidance document on testing procedures for pesticides in freshwater static mesocosm: A meeting of experts on guidelines for static field mesocosm tests. Workshop Report, Huntingdon, UK. SETAC-Europe, Bruselas, Bélgica. 46 pp.

SETAC-RESOLVE (1992) Workshop on aquatic microcosm for ecological assessment of pesticides: Sponsored by the SETAC Foundation for Environmental Education and RESOLVE (a program of the World Wildlife Fund). Wintergreen, Estados Unidos. $56 \mathrm{pp}$.

SEPÚLVEDA F, S MARÍN \& J CARVAJAL (2004) Metazoan parasites in wild fish and farmed salmon from aquaculture sites in southern Chile. Aquaculture 235: 89-100.

SOTO D \& R NORAMBUENA (2004) Evaluation of salmon farming effect on marine systems in the inner seas of southern Chile; a large-scale mensurative experiment. Journal of Applied Ichthyology 20: 493-501.

STARK J \& J BANKEN (1999) Importance of population structure at the time of toxicant exposure. Ecotoxicology and Environmental Safety 42: 282287.

SUTER G (1995) Ecological risk assessment. Lewis Publishers, London, Reino Unido. 538 pp.

TESTER P (1985) Effects of parental acclimation temperature and egg- incubation temperature on egg-hatching time in Acartia tonsa (Copepoda: Calanoida). Marine Biology 89: 45-53.

USEPA (1998) Guidelines for ecological risk assessment. Risk assessment forum U. S. Environmental Protection Agency. EPA/630/R-95/002F. Washington, Estados Unidos. $171 \mathrm{pp}$.

VALDOVINOS FS, R RAMOS-JILIBERTO, JD FLORES C ESPINOZA \& G LÓPEZ (2009) Structure and dynamics of pollination networks: The role of alien plants. OIKOS 118: 1190-1200.

VAN LEEUWEN C \& J HERMENS (1995) Risk assessment of chemicals: an introduction. Kluwer Academic Publishers, Dordrecht, Holanda. 374 pp.

WERNER EE \& JF GILLIAM (1984) The ontogenetic niche and species interactions in size-structured populations. Annual Review of Ecology and Systematics 15: 393-425.

WOOTTON JT (1994) The nature and consequences of indirect effects in ecological communities. Annual Review of Ecology and Systematics 25: 443-466.

ZAR JH (1999) Biostatistical analysis. Fourth edition. Prentice-Hall, New Jersey, Estados Unidos. 929 pp. 


\section{APÉNDICE}

Determinación del Riesgo Ambiental (modificado de Medina \& Encina 2003).

La determinación del riesgo ambiental puede ser definido como el proceso de caracterización y estimación de la probabilidad que efectos adversos hayan ocurrido, estén ocurriendo o vayan a ocurrir en la naturaleza como resultados de actividades antropogénicas (Suter 1995). Básicamente el riesgo ambiental de una sustancia se determina cualitativamente mediante la estimación de la razón entre la concentración ambiental esperada (PEC) y la concentración sin efecto ecológico (PNEC), a través de su cociente (RQ = PEC/PNEC). Un factor de evaluación o de incertidumbre (FI) puede también ser aplicado a los datos ecotoxicológicos existentes para así considerar el posible error en las mediciones, las variaciones en la manipulación de la información, la estocasticidad, la variabilidad natural del sistema, y los supuestos que se deben asumir frente a la falta de información en cada una de las etapas de la evaluación (Suter 1995, Norton et al. 1995). Los FI son constantes y se aplican según el nivel de incertidumbre que se tenga en la variable estudiada. Se considera que existe riesgo si el valor del cuociente es mayor que uno (PEC > PNEC). Por el contrario, si el valor del cuociente es menor que uno (PEC < PNEC) se considera que la presencia del agente contaminante, en el lugar estudiado y a las concentraciones esperadas, no representa un riesgo para el ecosistema.

El riesgo ambiental también puede ser evaluado utilizando un método probabilístico. En este, la incertidumbre es cuantificada considerando los supuestos hechos en la estimación y en la combinación de los parámetros involucrados (Suter 1995). A diferencia del método del cociente, el método probabilístico combina estocásticamente la distribución de posibles PEC con la distribución de los posibles efectos (PNEC), generando distintos valores del cociente de riesgo (Van Leeuwen \& Hermens 1995). Estas distribuciones son determinadas mediante modelos que integran algorítmicamente las distintas variables que intervienen en el nivel de exposición y en el efecto de un agente contaminante. 
